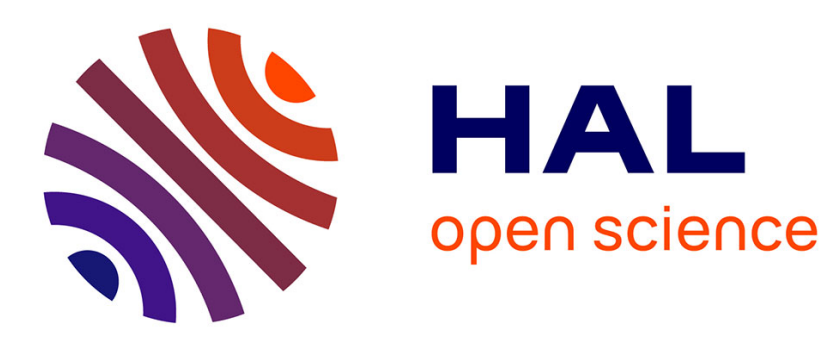

\title{
Optical Properties of Noble Metal Clusters from the Ab Initio Perspective
}

\author{
H.-Ch. Weissker
}

\section{To cite this version:}

H.-Ch. Weissker. Optical Properties of Noble Metal Clusters from the Ab Initio Perspective. Klaus Wandelt. Encyclopedia of Interfacial Chemistry, Elsevier, pp.546-558, 2018, 9780128097397. hal02002264

\section{HAL Id: hal-02002264 \\ https://hal.science/hal-02002264}

Submitted on 31 Jan 2019

HAL is a multi-disciplinary open access archive for the deposit and dissemination of scientific research documents, whether they are published or not. The documents may come from teaching and research institutions in France or abroad, or from public or private research centers.
L'archive ouverte pluridisciplinaire HAL, est destinée au dépôt et à la diffusion de documents scientifiques de niveau recherche, publiés ou non, émanant des établissements d'enseignement et de recherche français ou étrangers, des laboratoires publics ou privés. 


\section{Optical Properties of Noble Metal Clusters from the Ab Initio Perspective}

\section{Hans-Christian Weissker}

Aix-Marseille University, CNRS, CINaM UMR 7325, 13288, Marseille, France

and:

European Theoretical Spectroscopy Facility (ETSF)

www.etsf.eu ; http://etsf.polytechnique.fr

Address:

Campus de Luminy

Case 913

13288 Marseille Cedex 9

weissker@cinam.univ-mrs.fr

\section{Abstract:}

The peculiar optical properties of noble-metal clusters and nanoparticles are mostly governed by the localized surface-plasmon resonance (LSPR). The strong absorption arising from the LSPR in the visible and the near-UV parts of the spectrum of many clusters enables a multitude of applications. Here, we review the physical effects that dominate and influence the optical properties of noble-metal clusters in different size ranges, focusing in particular on the emergence phenomenon of the LSPR in gold clusters and on the presence of individual structure in the spectra which provide information on the cluster's quantum nature. Basic difficulties arising for experimental measurements of the optical properties are mentioned. We likewise discuss the different theoretical approaches suitable for the different size ranges.

Atomistic Time-Dependent Density-Functional Theory (TDDFT) is widely used for the description of noble-metal clusters of intermediate size, viz., between the molecule-like structures comprising but a few atoms and sizes of about $2 \mathrm{~nm}$. We discuss the basics of these ab initio TDDFT calculations, with a particular focus on the time-evolution approach. We discuss the present possibilities, strengths, and limitations of the method and compare briefly with the linear-response formalism. Finally, we discuss examples that highlight the strengths of the present TDDFT methods.

\section{Keywords:}

noble-metal, clusters, density-functional theory, time-dependent density-functional theory, TDDFT, ab initio, optical properties, surface-plasmon resonance, monolayer-protected clusters, single-particle measurements, linear response, time-evolution, RT-TDDFT, 


\section{Contents}

1 Metal Clusters and Nanoparticles: Their Role and History 3

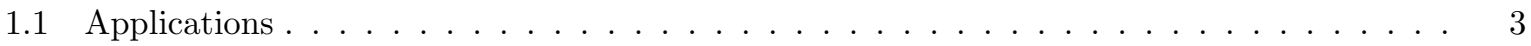

1.2 Size Ranges of Metallic Nanostructures . . . . . . . . . . . . . . . . . . . . . . . 3

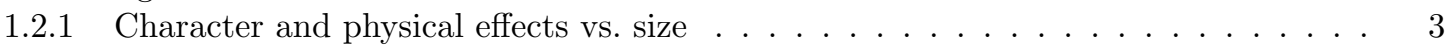

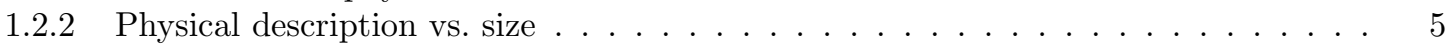

1.2.2.1 Classical description. . . . . . . . . . . . . . . . . . 5

1.2.2.2 Jellium-model-based descriptions. . . . . . . . . . . . . . . . . 6

1.2.2.3 Atomistic quantum description. . . . . . . . . . . . . . . . . . . . 6

1.3 Experiment ............................ 6

1.3.1 Ensemble measurements and their limitations . . . . . . . . . . . . . 6

1.3.2 Atomically precise monolayer-protected clusters . . . . . . . . . . . . 7

1.3.3 Measurement on individual NPs . . . . . . . . . . . . . . 7

2 TDDFT Calculations of Metal Clusters $\quad 7$

2.1 Optical Response from TDDFT: Time-Evolution Formalism . . . . . . . . . . . . 8

2.2 Comparison with Linear-Response Formalism . . . . . . . . . . . . . . . . . . . 10

2.3 RT-TDDFT: State-of-the Art, Virtues, and Limitations . . . . . . . . . . . . . . . . . . . 10

2.3 .1 Sizes . . . . . . . . . . . . . . . . . . . . . . . 10

2.3 .2 Geometry . . . . . . . . . . . . . . . . . . . . . . 11

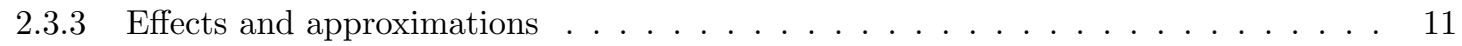

2.3.3.1 Exchange-correlation functionals . . . . . . . . . . . . . . 11

2.3.3.2 Time-evolution propagators . . . . . . . . . . . . . . . . . . 12

2.3.3.3 Interaction between electrons and ion cores . . . . . . . . . . . . . 12

2.3.3.4 Commonly neglected effects . . . . . . . . . . . . . . . . . . . . . 13

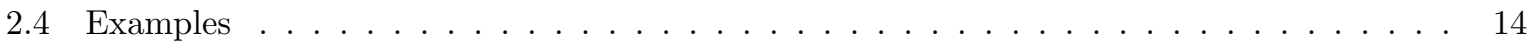

3 Further Reading $\quad 14$

4 References $\quad 16$ 


\section{Metal Clusters and Nanoparticles: Their Role and History}

\subsection{Applications}

Nanometric pieces of metal have properties which deviate decisively from the behavior of bulk metals. In many of them, localized surface-plasmon resonances (LSPR) occur which dominate the absorption of light in the visible range. As these depend on a number of parameters, including notably size, shape, chemical composition and configuration, and the nature of the surfaces surroundings, different colors can be obtained, for instance, in colloidal gold samples of different size. Historically, noble-metal nanoparticles, mostly gold, were employed as coloring agents. An outstanding example of such coloring is the Lycurgus cup which dates from probably the 4th century AD and is shown today in the British Museum. The presence of gold and silver nanoparticles, typically of the order of 50 to $100 \mathrm{~nm}$, endows the glass with a surprising dichroism: in reflection, the cup appears green, while when lighted from behind, it appears red [1]. In medieval brilliantly colored stained-glass church windows, noble-metal nanoparticles are likewise at play, although the real reason for the coloring effect must have escaped the makers of the glass, just as in the case of the Lycurgus cup.

Today, a plethora of realized or envisaged applications make use of the properties of noble metal nanoparticles (NPs) and clusters, many, but by no means all, based on their optical properties. The optical applications range from surface-enhanced Raman spectroscopy (SERS), biomolecule sensing, labeling of biomolecules, cancer therapy, and the plasmonic absorption enhancement in solar cells, to nanophotonics, and nanoplasmonics.

However, they also have a whole range of other surprising properties. Noble metal nanoparticles and clusters, mostly gold, have been in continuous use in biological and medical investigations over the past decade: bioconjugation chemistry, protein tagging, biomolecule labeling, inhibition of HIV fusion, and growth inhibition of bacteria are but a few of its applications. Finally, small gold clusters are catalytically active, unlike the rather inert bulk material. In addition to the direct catalytic effect, this opens the possibility of photocatalysis with noble metal clusters.

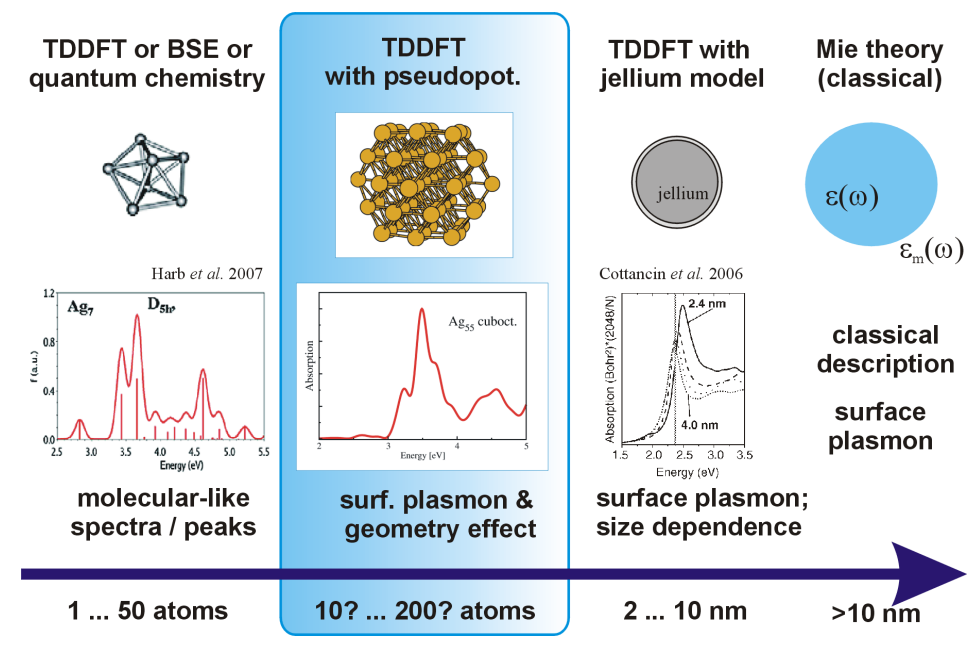

Figure 1 - Illustration of the different size ranges exhibiting different types of spectra, being dominated by different effects, and calling for different theoretical descriptions. The size-dependence of the LSPR in the inset is taken from Ref. [2].

\subsection{Size Ranges of Metallic Nanostructures}

Noble metal nanoclusters and nanoparticles span a very large range of sizes, from a few hundreds of nm to a few atoms, for an overview see Fig. 1 Their properties as well as the physical and chemical effects at play vary strongly with the size, as do, consequently, the theoretical means that enable their description and understanding.

\subsubsection{Character and physical effects vs. size}

Large noble metal nanoparticles (larger then, say, $10 \mathrm{~nm}$ ) can be seen as macroscopic pieces of bulk metal, with abrupt surfaces or interfaces. Their dielectric function corresponds to that of the bulk material, 
and screening is metallic, i.e., induced charges are restricted to the abrupt surfaces. Thus the interaction of light with the particle embedded in a medium is completely described in the framework of classical electrodynamics, assuming that the particle and the surrounding medium are continuous, homogeneous, and characterized by their respective dielectric function. The nanoparticles' properties will therefore reflect the fact that the electronic bands and the corresponding density of states are smooth.

In particular, collective oscillations of the (classical) electron density produce a smooth, broad feature in the nanoparticles' absorption spectra, the localized surface plasmon resonance (LSPR). This type of spectrum is shown in Fig. 1, a schematic representation of the oscillation in Fig. 2. No quantum effects need be evoked in the understanding of these systems, nor is there any notion of inhomogeneity at the atomistic level.

Building on the work of L. Lorenz and J. C. Maxwell-Garnett, Gustav Mie published in 1908 his famous article [3] "Beiträge zur Optik trüber Medien, speziell colloidaler Metallösungen" ("Contributions to the optics of turbid media, particularly of colloidal metal solutions") in the Annalen der Physik. Using classical electrodynamics, i.e., the solution of Maxwell's equations, his seminal work calculates the scattering of a plane electromagnetic wave by a spherical metal particle. It became so influential that even today, the (localized) surface plasmon is often referred to as Mie plasmon.
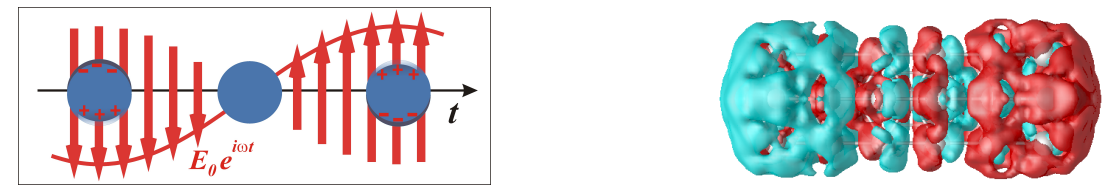

Figure 2 - Left panel: schematic of the classical picture of the SPR as a density oscillation. Right panel: Snapshot of the density dynamics showing the longitudinal charge oscillation in a 37-atom Ag nanorod (cf., Ref. [4]).

When going to smaller systems (smaller then, say, $10 \mathrm{~nm}$ ), quantum effects start to play a role. In particular, the classical assumption of abrupt interfaces does not remain valid but an effective treatment of fields and densities is still possible. In the case of noble metals in vacuum, a blue shift of the surface plasmon frequency is observed with decreasing cluster size, unlike in alkali clusters which show a red-shift. This size dependence of the plasmon resonance results from a number of effects [5-7], of which two are especially important: a spill-out of the conduction $s$ electrons beyond the classical particle radius leads to a red shift with respect to the classical description, and a reduced $s$ - $d$ screening in the surface region in the case of the noble metals leads to a blue shift. The interplay of the two counteracting effects produces the respective size dependence because the surface region becomes relatively more important when the size decreases. For even smaller clusters, the atomistic structure will become important [8].

On the lower end of the size range, very small clusters, comprising a few atoms to a few dozen atoms, are essentially molecules which exhibit absorption spectra with individual transitions that reflect the discrete electronic structure. Clearly, these small clusters are many-particle quantum systems that are not amenable to classical concepts, even though part of the effects may remain similar to classical behavior [9].

With increasing size, these discrete electronic levels become closer until they form the bands that characterize the bulk metals. The resulting optical absorption spectra are gradually becoming smooth, showing (like in $\mathrm{Ag}$ ) or not showing (like in $\mathrm{Au}$ or $\mathrm{Cu}$ below about $2 \mathrm{~nm}$ ) the LSPR.

This transition region - from clusters of a few dozen atoms to diameters of 2 or $3 \mathrm{~nm}$ where the discrete spectra give way to smooth spectra, with or without LSPR - is the most interesting because here the collective and classical effects combine with the quantum effects that witness the quantum information of the electronic structure on the one hand (quantum size effects that distinguish the NP from the bulk material) and, on the other hand, the interplay between spill-out effects at the NP surface and the polarizability of the d electrons. The SPR may be fragmented, and the inhomogeneity at the atomistic level may have a strong effect.

The transition size range is likewise the least understood because on the one hand, it is extremely difficult to create and characterize the respective systems with the precision that brings out individual features (cf., section 1.3), and on the other hand because this size range has not been accessible to atomistic quantum calculations until recently. The role of the electronic structure, the intricate effects 
that influence the optical response, and the influence of substrates and ligands remain poorly understood.

It needs to be emphasized that the emergence of the LSPR and the transition from discrete to smooth spectra are two distinct phenomena, although of course related. Their understanding is a formidable challenge for the theoretical treatment. In general, the two principal questions connected with this transition region can be formulated as follows:

(1) Quantum information. How does the development of smooth electronic bands from the discrete states of the strongly localized tiny clusters happen, what are the physical effects that determine this transition, and how can they be described properly?

For instance, it has been shown only very recently that the $\mathrm{Au}_{144}(\mathrm{SR})_{60}$ cluster still shows spectra of high information content (many individual peaks) which are not yet, however, covered by a strong LSPR [10]. This finding, shown in Fig. 4, is highly important because it was generally believed that clusters of this size necessarily have already smooth spectra of low-information content [11].

(2) Emergence of surface-plasmon resonance. What are the precise conditions and mechanisms that produce the plasmon emergence in some materials with increasing size, or the presence of plasmonic excitations down to very small sizes?

Gold and silver have almost identical lattice constants. Moreover, the valence electron structure is similar, with a complete $\mathrm{d}$ shell and one s electron $\left(\ldots 4 \mathrm{~d}^{10} 5 \mathrm{~s}^{1}\right.$ for silver, $\ldots 5 \mathrm{~d}^{10} 6 \mathrm{~s}^{1}$ for gold) and they may be alloyed in the bulk at any composition. However, the optical properties are the two noble metals are strongly different, as it can already be seen from the different colors of the bulk metals. This is due to the fact that the $d$ bands lie at different energies below the Fermi energy: at around $2 \mathrm{eV}$ for gold, and at around $4 \mathrm{eV}$ for $\mathrm{Au}$ (and for $\mathrm{Cu}$ ). This means that the LSPR in silver clusters is largely decoupled from the interband transitions i.e., transitions from the $\mathrm{d}$ band into states above the Fermi energy. In gold and copper, on the other hand, the LSPR lies within a background of interband transitions, which leads to its broadening and damping, up to the point of making it disappear for sizes below about $2 \mathrm{~nm}$ for gold.

Any effects that influence the LSPR energy influences, therefore, the emergence of the LSPR. This may be the LSPR blue shift with decreasing size, which is mainly responsible for the disappearance of the LSPR in small gold clusters. However, if a means is found to lower the LSPR energy, the LSPR may emerge in very small clusters as well. This is the case, for instance, in the case of nanorods where a strong aspect-ratio-dependent red-shift produces strong resonances for high-aspect-ratio rods $[9,12]$.

\subsubsection{Physical description vs. size}

As the main physical effects depend on the sizes of the clusters, so do the theoretical means that enable their description.

\subsubsection{Classical description.}

For large noble metal nanoparticles, the resonances observed in absorption spectra have been interpreted as collective excitations of the electrons, cf., Fig. 2. Classical Mie theory can be used to describe them. There, the interaction of light with the particle embedded in a medium is described in the framework of classical electrodynamics, assuming that the particle and the surrounding medium are continuous, homogeneous, and characterized by their respective dielectric function, taken usually from the corresponding unperturbed bulk materials. In this description, the interfaces between nanoparticle and surrounding matrix are abrupt, and the induced charges are restricted to these abrupt surfaces.

Going to smaller systems, below maybe $10 \mathrm{~nm}$, these conditions do not sufficiently represent the physical situation anymore - quantum effects start to play a role. In particular, the idea that charges are only induced at the abrupt boundaries needs to be abandoned. For instance, for small clusters in vacuum, classical (local) Mie theory does not show any change in SPR frequency upon changing size. However, the experimental results do [13].

In recent years, great theoretical interest has been focused on this field, and effective treatments, such as the hydrodynamical Drude $[14,15]$ and the quantum corrected [16] models, have been proposed. These 
methods aim to fill the mesoscopic gap between Maxwell's equations and condensed matter theory. They are designed to describe the electrodynamic response of macroscopic structures (containing millions of electrons) significantly affected by electronic quantum effects taking place at their microscopic features.

\subsubsection{Jellium-model-based descriptions.}

For smaller sizes (below about $10 \mathrm{~nm}$ ), quantum effects become important. The energy, intensity, and width of the LSPR are determined by the intricate interplay between spill-out effects of the conduction electrons and the polarizability of the $d$ electrons. For simple metals, the jellium-background model has been applied in which the ion cores are represented by a homogeneous background [17]. In the noble metals with their polarizable d electrons, this approach is not sufficient. However, the d-electrons can be treated via an appropriate dielectric function. For diameters of about 10 nanometers, general trends and - in most cases - quantitative predictions, are in good agreement with experiments [2]. Due to its simplicity (the discrete ionic structure is disregarded) absorption cross-sections of clusters containing up to 40000 conduction electrons can be computed. However, it is evident that any interface or interaction effects on a atomistic level (including charge transfer) will not be described.

\subsubsection{Atomistic quantum description.}

On the lower end of the size range, very small molecule-like clusters have been treated theoretically with quantum-chemical methods, we only name configuration-interaction (CI) and the linear response equation-of-motion coupled-cluster (EOM-CC) method.

Time-dependent density functional theory (TDDFT) has been used on small and intermediate-sized noble metal clusters [18]. In the size-range of up to about 150 or 300 atoms, TDDFT has become the method of choice for the atomistic quantum description of clusters. The three TDDFT most commonly used for noble-metal clusters of intermediate size are the octopus code [19], the ADF code [20] and GPAW [21]. These quantum calculations take into account the electronic structure, with an explicit treatment of both s- and d-electrons. In these calculations, the ion cores are either described by pseudopotentials or using localized orbitals. In this way, the inhomogeneity of the metal on the atomistic level is taken into account.

The TDDFT calculations necessarily apply approximations (cf., below in Sec. 2.3.3) which must be well controlled. This includes comparison with experiment. However, one of the reason for the unavailability of precise comparisons in many cases is the absence of reliable benchmark results for intermediate sizes. The reasons for this are explained in the following section.

\subsection{Experiment}

To understand the physics of the clusters, well-controlled characterization of well-controlled clusters is necessary. Measurements need to connect the structural information like size and geometry with measured properties. Herein, we do not give a comprehensive overview of experimental methods. Instead, we point out a few issues that are important in the present context.

A lot of knowledge has been obtained from gas phase experiments. Plasmonic absorption in large, as well as discrete transitions in small, metal clusters have been evidenced. Gas-phase experiments have produced results concerning the size-dependence of the LSPR energy [13, 22]. However, the interpretation of many experiments remains complex. For instance, even in an experiment with perfectly size-selected clusters, the geometries of all the clusters need not be identical.

The situation becomes even more complex if the metal clusters in question are no longer in vacuum but put into contact with an environment, be it by deposition on a surface, embedding it in a solid matrix or by covering it with organic surfactants. This complexity is, however, the price one has to pay in order to stabilize the inherently unstable metal cluster against thermal or chemical decomposition in view of a possible exploitation of its tunable physical properties in technological applications.

\subsubsection{Ensemble measurements and their limitations}

Ensemble measurements typically suffer from both, differences in the local environment (interfaces, ligands, oxidation, matrix...), and the inhomogeneity of the samples (size distributions; differences in com- 
position; charge states; orientations). As a consequence of the resultant inhomogeneous broadening of the spectra, individual fine structure is usually not visible. This leads to the absence of precise benchmark results for intermediate sizes and severely limits the comparison with theoretical results. Instead, broad, smooth spectra are obtained as shown in Fig. 1 [2].

There are essentially two ways out of this problem as discussed in the following section.

\subsubsection{Atomically precise monolayer-protected clusters}

Monolayer-protected clusters like the aforementioned $\mathrm{Au}_{144}\left(\mathrm{SCH}_{3}\right)_{60}$ present a possibility to study atomically controlled clusters and compounds without size distributions, unlike in the case of matrix-embedded clusters. Particularly stable compounds can then be separated and studied. Samples may be prepared in solution or in glassy matrices and optically characterized. For instance, the $\mathrm{Au}_{144}(\mathrm{SR})_{60}$ cluster compound has been found to exhibit individual spectral peaks only very recently [10] (see Fig. 4; it was believed for a long time to be as large as to have smooth spectra.) Moreover, STEM Electron Diffraction and High-Resolution Images can be used to obtain precious structural information. In addition, some of the systems have been crystallized so that a total structure determination using x-ray scattering has been possible [23].

\subsubsection{Measurement on individual NPs}

The second possibility to escape the problems of inhomogeneous broadening are single-particle measurements. One option is electron energy-loss spectroscopy (EELS) in the transmission electron microscope. For the interesting size-range between 1 and a few $\mathrm{nm}$, such measurements have recently become feasible [24] but have produced results that are partially in contradiction with the bulk of previous studies [13]. It is practically certain that the interaction of the NPs with the matrix plays an important role in this discrepancy. This type of measurement is expected to obtain increasingly precise results in the future.

An alternative for optical single-particle measurements is the spatial modulation spectroscopy technique [25].

\section{TDDFT Calculations of Metal Clusters}

In principle, the problem to be solved is that of an interacting many-electron system moving coupled to the movement of the ion cores. The first step to simplify is to separate the motion of cores and electrons using the Born-Oppenheimer approximation which is based on the fact that the electrons move much faster than the ions. This results in the interacting electrons moving in the external field of the ion cores plus any additional fields like, in particular, laser fields that excite the system.

As mentioned before, density-functional theory (DFT) and its time-dependent version, time-dependent density-functional theory (TDDFT) have become the approach(es) most commonly used in quantum calculations of metal clusters. Other methods exist to calculate electronic excitations. In particular, many-body perturbation theory has been applied to very small metal clusters [26] and compared with TDDFT calculations. The relation between many-body perturbation theory and TDDFT is comprehensibly reviewed by Onida et al. [27]. For very small clusters, high-level quantum-chemistry calculations provide precious benchmarks to test the performance of particular approximations.

Ground-state DFT rests on the Hohenberg-Kohn theorem which demonstrates the one-to-one correspondence between a given external potential (up to an added constant) and the ground state electron density of the system. The density that minimizes the Hohenberg-Kohn energy functional is the true ground-state density of the system. Practical calculations can be done using the Kohn-Sham formalism in which a system of equations for non-interacting electrons is solved which was derived to give the same total energy and electron density as the full, interacting many-body Schrödinger equation would. The approach is limited to the ground state. In particular, the so-called Kohn-Sham energies which appear to be similar to the eigenenergies of a Schrödinger equation do not have a clear physical meaning (except for the highest occupied state). Considering them nonetheless as excitation energies - i.e., band energies in solids as measured in photo-emission - one runs into the well-known "band-gap problem" of DFT: the energy gap of solids is usually underestimated. This is on the one hand due to the approximations used for the exchange-correlation functional, but on the other hand even the exact functional would not yield correct excitation energies because DFT is a ground-state theory.

To include the description of excitations, the time-dependent version of DFT, TDDFT, can be employed. The Runge-Gross theorem [28] establishes a correspondence between a time-dependent scalar external field and the time-dependent density. The steps leading to this result are more involved than in 
the static case, and the limitations are more stringent. In particular, for extended systems, the theorem is only valid for periodic densities and external fields, which means that the response to a uniform electric field cannot be treated [29]. However, for very small clusters that can be treated using TDDFT methods, we need not be concerned with these limitations.

Important for actual calculations, a time-dependent Kohn-Sham scheme can be devised in which the time evolution of the time-dependent density of the true, interacting system can be reproduced by a non-interacting system. In practice, we start with a system that for $t<t_{0}$ is in its ground state, and we write the time-dependent scalar external potential as

$$
v(\mathbf{r}, t)=v_{0}(\mathbf{r})+v_{1}(\mathbf{r}, t) \theta\left(t-t_{0}\right)
$$

where $\theta$ is the step function. In this situation, the Hohenberg-Kohn theorem applies to the initial state $\Psi_{0}$. This initial state $\Psi_{0}$ is a single Slater determinant of the N Kohn-Sham orbitals $\phi_{j}^{0}(\mathbf{r})$ which are the self-consistent solutions of the static Kohn-Sham equation

$$
\left[-\frac{\nabla^{2}}{2}+v_{s}^{0}\left[n_{0}\right](\mathbf{r})\right] \phi_{j}^{0}(\mathbf{r})=\epsilon_{j} \phi_{j}^{0}(\mathbf{r})
$$

with the ground state density

$$
n_{0}(\mathbf{r})=\sum_{j=1}^{N}\left|\phi_{j}^{0}(\mathbf{r})\right|^{2}
$$

and the static Kohn-Sham potential

$$
v_{s}^{0}\left[n_{0}\right](\mathbf{r})=v_{0}(\mathbf{r})+\int d^{3} \mathbf{r}^{\prime} \frac{n_{0}\left(\mathbf{r}^{\prime}\right)}{\left|\mathbf{r}-\mathbf{r}^{\prime}\right|}+v_{x c}^{0}\left[n_{0}\right](\mathbf{r}),
$$

where $v_{0}(\mathbf{r})$ is the external potential, including, in particular, the potential created by the ion cores and thus representative of the structure under study. The second term expresses the electrostatic electronelectron interaction, whereas the exchange-correlation potential $v_{x c}\left[n_{0}\right](\mathbf{r})$, which is a functional of the density, describes the exchange and correlation effects.

Let us assume that the system is in its ground state before $t=t_{0}$. Starting immediately after $t_{0}$, the system evolves under the influence of the time-dependent potential $v_{1}(\mathbf{r}, t)$. This is described by the time-dependent Kohn-Sham equations

$$
\left[-\frac{\nabla^{2}}{2}+v_{s}[n](\mathbf{r}, t)\right] \phi_{j}(\mathbf{r}, t)=i \frac{\partial}{\partial t} \phi_{j}(\mathbf{r}, t),
$$

the initial conditions are presented by the ground-state Kohn-Sham wave functions,

$$
\phi_{j}\left(\mathbf{r}, t_{0}\right)=\phi_{j}^{0}(\mathbf{r})
$$

and the time-dependent density is

$$
n(\mathbf{r}, t)=\sum_{j=1}^{N}\left|\phi_{j}(\mathbf{r}, t)\right|^{2} .
$$

Equations 5 and 7 together with the initial conditions eq. 6, provide a scheme that is in principle exact and which provides the full dynamics of the time-dependent Kohn-Sham system and, therefore, of its time-dependent density which is equal to that of the full interacting system. In particular, this provides the time-dependent dipole moment of the system, which will in turn provide the optical response.

However, to obtain a practically useful scheme, a number of additional steps need to be carried out which include certain approximations.

\subsection{Optical Response from TDDFT: Time-Evolution Formalism}

To calculate the optical response using TDDFT, different formalisms exist. In particular, linear-response TDDFT calculates the response to a weak external field that is treated as a perturbation [27, 30].

A complementary approach is the time-evolution formalism as introduced by Yabana and Bertsch [31]. This approach is not restricted to the linear regime and has been used for systems in strong laser fields. 

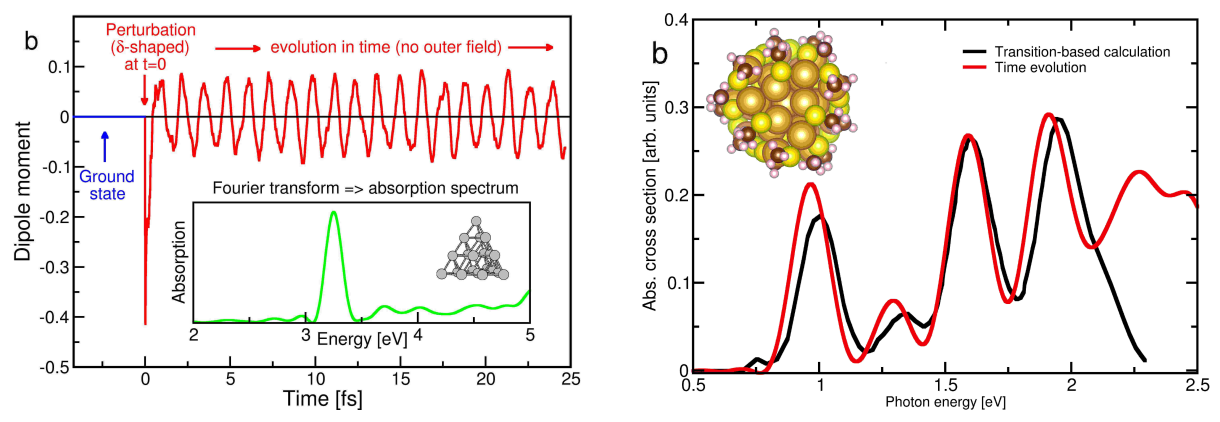

Figure 3 - (a) Schematic presenting TDDFT real-time calculation as introduced by Yabana et al. [31], applied to a 20-atom Ag tetrahedron. After a ground-state calculation a perturbation is applied, after which system evolves freely. The absorption spectrum is obtained as the Fourier transform of the time-dependent dipole moment. (b) To demonstrate the equivalence with the transition-based Casida approach (LR-TDDFT), we compare a calculation with the octopus code, using the present numerical set-up, with a calculation from Ref. [32] for a $\mathrm{Au}_{38}$ cluster.

However, by choosing a perturbation that is weak enough so as to ensure the linear dependence between perturbation and response, linear absorption spectra can be calculated.

From the start, we apply the dipole approximation: the system is assumed to be much smaller than the wavelength of the perturbing electrical field, i.e., a laser. The electrical field varies only slightly over the length scale of the system, and it is a good approximation to neglect its spatial dependence altogether. The particle "sees" only a spatially constant field with a time dependence $e^{i \omega t}$. This condition is well realized for the clusters treated in the present work and for light in the visible range. In the dipole approximation, the interaction with a field in $z$ direction is

$$
V(\mathbf{r}, t)=e z E(t) \hat{e}_{z} .
$$

Assuming for simplicity that the total induced dipole $p(t)$ is parallel to $\mathbf{E}(t)$,

$$
p(t)=\int_{-\infty}^{\infty} d t^{\prime} \alpha\left(t-t^{\prime}\right) \mathbf{E}\left(t^{\prime}\right)
$$

where $\alpha(\tau)$ is the time representation of the polarizability of the system (in the general case, it would be the tensor $\left.\alpha_{i j}(\tau)\right)$. This can be Fourier-transformed into

$$
p(\omega)=\alpha(\omega) \mathbf{E}(\omega) .
$$

For a monochromatic laser field polarized along the $z$ direction, $E z \sin (\omega)$, the $z$ component of the dipole becomes

$$
p(t)=\int d^{3} \mathbf{r} z n(\mathbf{r}, t) .
$$

After Fourier transform, and by virtue of eq. 10, this becomes

$$
\alpha(\omega)=-\frac{2}{E} \int d^{3} \mathbf{r} z \delta n(\mathbf{r}, \omega),
$$

the imaginary part of which in turn is directly linked to the absorption cross section $\sigma(\omega)$ by

$$
\sigma(\omega)=-\frac{4 \pi \omega}{c} \Im \alpha(\omega) .
$$

This shows that in order to calculate the optical absorption, it is enough to calculate the Fourier transform of the time-dependent dipole moment. However, the question remains as to what excitation should be chosen.

Yabana and Bertsch [31] have considered an external field the time dependence of which is a delta function in time,

$$
E^{\text {delta }}(t)=I \delta(t),
$$


where $I$ is the magnitude of the field. The Fourier transform of this field, $E(\omega)=I$, contains all frequency components equally. Consequently, we find for a system excited from its (unpolarized) ground state

$$
\alpha^{\text {delta }}(\omega)=\frac{1}{I} \int_{0}^{\infty} d t p^{\text {delta }}(t) e^{i \omega t} .
$$

In the practical implementation, one wants to avoid working with the $\delta$ function because it is ill adapted to work with the discretized time. Yabana and Bertsch showed that the multiplication of the wave functions at $t=t_{0}$ with a phase factor $\exp \{-i I r\}$ is a practically viable form of applying the $\delta$ kick. This is the form of the calculation as used, for instance, in the code octopus [19].

\subsection{Comparison with Linear-Response Formalism}

The time-evolution formalism (often called RT-TDDFT, for "real time") is complementary and physically equivalent to the linear response formalism (LR-TDDFT). In the latter, the response of the electronic system to a perturbation is calculated $[27,30]$. The respective equations are solved such as to include only the linear part of the series expansion of the effects of a perturbation. By contrast, the time-evolution formalism has no such restriction and has been conceived to treat both non-linear optical properties and clusters in very strong laser fields. However, our choice of the time-evolution formalism is based on other reasons:

The propagation of the time-dependent Kohn-Sham wave functions is only necessary for the occupied states, unlike in LR-TDDFT calculations, which must include a large number of empty states. This makes the RT-TDDFT calculations numerically more affordable for large systems, in particular with respect to memory requirements.

Another advantage of the time-evolution formalism is that the propagated wave functions provide directly the motion of the electron density. This allows to study, e.g., for noble-metal clusters, the validity of the commonly presented picture of the LSPR as a charge oscillation, shown in Fig. 3.

A disadvantage of the time-evolution formalism is that the origin of features in the absorption spectrum is not easily determined, unlike in the case of LR-TDDFT where at least the contribution of the participating Kohn-Sham transitions are easily found. The spatially resolved Fourier transform of the total time-dependent density provides a partial remedy to this shortcoming, albeit only in terms of spatial contributions. A strict way of obtaining this information is the projection of the time-dependent wave functions onto the ground state wave functions. This provides the link between the "transition picture" in which the optical excitations are discussed in terms of transitions between stationary states, and the time-evolution formalism in which the same excitations are described by the time-evolution of the occupied Kohn-Sham states. A good example of it can be found in Ref. [33] for jellium clusters.

Another interesting approach has been published on arxiv recently by Rossi et al. [34]. The authors use time evolution and a Kohn-Sham decomposition with certain approximations. It will be interesting to see the virtues of this approach in the future.

\subsection{RT-TDDFT: State-of-the Art, Virtues, and Limitations}

\subsubsection{Sizes}

One of the problems of the atomistic TDDFT calculations is the size of the clusters; in many cases the memory requirements but also the availability of $\mathrm{CPU}$ time decide about the sheer feasibility of a given study. The large number of electrons (11 per atom) renders the calculations cumbersome compared to similar calculations for semiconductor nanocrystals or biological systems. However, this field is developing fast, with a growing number of groups participating. Over the last few years, calculations of clusters of around 150 atoms (diameters just below $2 \mathrm{~nm}$ ) have become rather routine, with exceptional calculations of more than 300 gold atoms.

Moreover, many technical and algorithmic developments (concerning machines but also software) have increased the accessible size range. One step in this direction is the use of the PAW method instead of norm-conserving pseudopotentials, as in the code GPAW [21].

In addition, some theoretical developments of approximations allow for a reduction of the numerical effort compared to a "standard" TDDFT calculation. However, in some cases, the quality of the results is not immediately clear. Nobusada et al. have developed a code which can calculate the spectra of clusters of up to 1500 noble-metal atoms [35]. Stener and Fortunelli have recently published a new approximation to reduce the numerical effort [36]. A last development that should be mentioned is the "Orbital-free TDDFT." In the case of clear plasmonic resonances, this method seems to fare well, but the details of 
other parts of the spectra and the deduced density dynamics seem to be less well described, as shown for Na clusters [37].

\subsubsection{Geometry}

The atomistic TDDFT calculations need precise knowledge of the structures - the position of every atom needs to be known. To find them is far from trivial.

Very large nanoparticles remain in their crystallographic bulk structure (fcc, in the case of the noble metals), and their overall shape is determined by a minimization of the different surface energy contributions. (Wulff construction for free nanoparticles and Wulff-Kaishew for nanoparticles on surfaces.)

For smaller particles, non-crystalline structures become possible. In particular, five-fold symmetries, which are ruled out in bulk materials, start to occur, which is particularly visible in decahedral and icosahedral clusters or nanoparticles.

Finally, very small clusters can take diverse forms that have lost any connection with the respective bulk structures $[38,39]$.

Two main problems exist for the simulation: (i) the determination of the clusters' energy for a given geometry, and (ii) the sampling of the very large parameter space defining the geometries.

(i) for the calculation of energies, a large number of empirical and semi-empirical potentials have been devised [38, 39]. DFT is also being used, although even this does not always lead to unequivocal results because the use of different exchange-correlation functionals may lead to different structures.

(ii) For the determination of the structure (once the calculation of the energies according to (i) decided), one has likewise a plethoric choice [38,39]. This includes molecular dynamics and simulated annealing, Monte-Carlo methods both in free geometry and on fixed lattices, basin-hopping methods and, last but not least, genetic algorithms. The latter have been combined, in particular, with densityfunctional theory calculation of energies [40]. While this leads to very good results, it is restricted to very small systems.

In all these methods, there is hardly any guarantee that the global minimum structure has been found. It should be verified that the structure is at least in a local minimum of the potential energy surface. A common test of (meta-)stability is the calculation of vibrational modes; if imaginary frequencies occur, the structure is not in a true minimum but rather on a saddle-point-like position on the total energy surface.

Finally, for a number of monolayer-covered clusters, experimentally structure-determined cases are available. Crystallized samples can be treated using x-ray diffraction or also electron diffraction. In these cases, calculations can start from the rather precise experimental coordinates and than relax the structures using DFT total energy calculations [23].

\subsubsection{Effects and approximations}

TDDFT calculations necessitate a number of approximations which must be well adapted to the problem under study. Certain effects are neglected in most calculations. Obviously, their relative importance will vary for different systems, different sizes, and different observables.

\subsubsection{Exchange-correlation functionals}

For the solution of the time-dependent Kohn-Sham equations 5, an approximation of the time-dependent exchange-correlation functional $v_{s}[n](\mathbf{r}, t)$ is needed (along with the static $v_{s}^{0}\left[n_{0}\right](\mathbf{r})$ in eq. 2). For the time-dependence, very often the most simple approximation is used, called adiabatic: the time-dependent $v_{s}[n](\mathbf{r}, t)$ is approximated by that of the ground state, with $n(\mathbf{r}, t)$ replacing the ground state density,

$$
v_{\mathrm{xc}}(\mathbf{r}, t)=\left.v_{\mathrm{xc}}\left[n_{0}\right](\mathbf{r})\right|_{n_{0} \rightarrow n(\mathbf{r}, t)} .
$$

Clearly, this approximation excludes memory effects. However, in many cases, this approximation gives very reasonable results. The role of energy-dependent kernels, which are the functional derivative of the exchange-correlation functional with respect to the density, occurring in linear-response TDDFT, is discussed, e.g., by Ullrich [29]. 
In general, the construction of good exchange-correlation functionals is a central problem of DFT and TDDFT because this is the term that contains all the complicated many-body interactions. What a good approximation is may vary from one situation to another. In very small, and thus strongly localized molecules and clusters, the short-range interactions need to be well described, unlike in extended bulk systems where the long-range interactions play an equally important role [41]. Moreover, for the noble metals, the interaction between the s and the d electrons must be very well described.

Many TDDFT calculations use (adiabatic) local or semi-local density approximations, i.e. either LDA (local-density approximation) or standard GGA (generalized gradient approximations) density functionals. However, these produce an incorrect asymptotic form which leads to errors in optical properties. The potential of pure density functionals decays to zero exponentially rather than as $-1 / r$ at large $r$. Various asymptotically corrected potentials have been constructed and added to a GGA functional. For examples, LB94 [42] and SAOP [43] are local potentials which give the correct $-1 / r$ behavior, but they vanish at infinity. To give a more suitable description of charge-transfer excitations in the adiabatic linear-response approximation, a long-range correction may be applied to the exchange functional. In range-separated hybrid functionals [44], the Coulomb operator is split into two parts: a short-range term evaluated with the exchange potential from DFT, and a second term, the long-range part, evaluated with the nonlocal Hartree-Fock (HF) exchange. It has the drawback to lose the local properties of the potential and needs much more computational resources. Rabilloud et al. have recently assessed the accuracy of several longrange corrected density functionals for calculating the absorption spectra of small silver clusters [45]. For very small silver clusters, excellent agreement with experiment has been obtained, unlike in the case of very small gold clusters.

Many systematic studies of the virtues and limitations of different functionals have been carried out [46]. Important for practical calculations is, in particular, the existence of the library LIBXC [47] which allows for easy use of many different functionals for instance in calculations with the octopus code.

Many studies of clusters of intermediate size consider mostly general effects, with comparison to experiment - until now - being qualitative rather than quantitative. Very often, the simple functionals GGA (PBE), LDA, or LB94 have been used. Naturally, the quality of the description must be verified. Sinha-Roy et al. have compared GGA (PBE) spectra of silver rods of different size and aspect ratio with spectra using the range-separated hybrid LC-M06L $[48,49]$ that gives the excellent results for small silver clusters [45] and is, therefore, considered as benchmark. GGA (PBE) spectra are in very good agreement with LC-M06L as long as there is a clear plasmonic resonance that is well separated from interband transitions from the $\mathrm{d}$ electrons. However, when the coupling with the $\mathrm{d}$ electron excitations becomes strong, the quality of the spectra degrades strongly [9]. By contrast, for small gold clusters, a good agreement between TDDFT spectra and experiment has not yet been achieved. Nonetheless, the PBE spectra calculated for the monolayer-protected $\mathrm{Au}_{144}(\mathrm{SR})_{60}$ are in decent agreement with experiment [10].

\subsubsection{Time-evolution propagators}

A numerical algorithm is needed to propagate the Kohn-Sham wavefunctions of eq. 5 . In practice, one discretizes time with a time step $\Delta \tau$. If the wave functions are known at a time $\tau_{n}$, the wave functions at the subsequent time step $\tau_{n+1}$ can be written formally as

$$
\phi\left(\tau_{n}+\Delta \tau\right)=\hat{U}\left(\tau_{n}+\Delta \tau, \tau_{n}\right) \phi\left(\tau_{n}\right)
$$

where $\hat{U}\left(\tau_{n}+\Delta \tau, \tau_{n}\right)$ is the time-evolution operator that propagates the orbitals by one step $\Delta \tau$. In practice, the time evolution can be implemented numerically in different ways where stability is an important criterion. An application-oriented study of different propagators has been carried out by Castro et al.. [50].

\subsubsection{Interaction between electrons and ion cores}

The interaction of the electrons with the atom cores needs to be described. In most of the modern calculations one uses the fact that only the valence electrons close to the Fermi energy contribute to chemical binding and to the optical response. The more strongly bound electrons in the filled lower shells can be treated as unchanging under the processes we are interested in, which is done in the frozen core approximation. In addition, they can then be described using the pseudopotential approximation $([51,52])$ which constructs a rather smooth potential representing the combined potential of the atomic cores and the frozen electrons. Clearly, the choice as to which electrons are included into the pseudopotential and which are treated explicitly depends on the atom and on the properties one wants to calculate. Over the 
last few years, Blöchl's Projector-Augmented Wave method (PAW), has superseded the pseudopotential method. It produces numerically less demanding, softer potentials, and the true wavefunctions close to the center of the atom can be recovered from the pseudoized calculation because it is built on a linear transformation rather than a rather arbitrary approximation as in the case of the pseudopotentials [53].

\subsubsection{Commonly neglected effects}

Spin polarization. In most studies of intermediate-sized noble metal clusters, spinless calculations are performed where the spin-polarization is neglected, which is justified for noble-metal clusters, which are non-magnetic with very few somewhat exotic exceptions. Electron spin is taken into account by the fact that each state if occupied by two, not one, electrons.

Spin-orbit coupling. In most TDDFT calculations, the relativistic corrections for gold and silver are accounted for at a scalar level through the use of relativistic pseudopotentials (or the so-called ZORA approximation). This is essential, in particular for gold. However, most calculations neglect spin-orbit (SO) coupling. While the latter is not important in silver, it may play a significant role in gold. SO coupling leads to changes in the spectra of very small clusters $\left(\mathrm{Au}_{n}, n=2-9\right)$, including shifts of some main peaks and specially a dispersion of the oscillator strengths resulting into a broadening and damping of the optical response [54]. By contrast, SO coupling effects on the spectrum of $\mathrm{Au}_{20}$ were found to be relatively small [55]; the SO coupling effects are somewhat less important for large and compact systems.

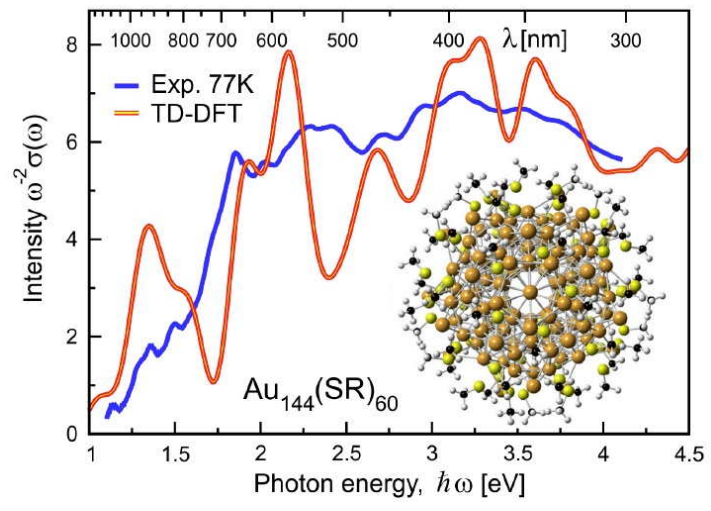

Figure 4 - Optical spectra for the ubiquitous icosahedral gold clusters, $\mathrm{Au}_{144}(\mathrm{SR})_{60}$. Theory (red) and experiment (blue). Plotted is the optical absorption cross-section frequency-weighted to match the optical-absorbance function. Figure taken from [10] and its Supplementary Material.

Temperature effects are neglected in most calculations, although they cause a significant broadening of spectral features - the individual structures in the visible range of low-temperature spectra of $\mathrm{Au}_{144}(\mathrm{SR})_{60}$ are almost entirely washed out at room temperature [10]. To include temperature effects on the optical spectra of nanostructures, several methods can be employed. The direct inclusion of the electron-phonon coupling in the framework of $a b$ initio calculations [56] is unlikely to be practicable for clusters of intermediate size soon. However, explicit calculation of lattice-vibrations of clusters and nanocrystals using Born-Oppenheimer molecular dynamics with a subsequent averaging to obtain the desired quantities has been employed in a number of studies, for instance, on small sodium clusters [57].

Interface and matrix effects. The environment of the noble-metal clusters plays an important role for many properties. These effects act on different levels. Many of them have been explicitly treated for molecules or very small molecular clusters. However, for clusters of intermediate size, most of the effects enumerated here present challenges for future work.

1) Chemical bonding and the resulting charge transfer between NP and surrounding evidently influence the optical properties decisively. This applies in particular to the wet-chemically produced mono-layerprotected clusters. Here, very often the surface structure itself is entirely different from what it would be for a bare cluster. In this category of cluster-interface interactions fall also oxidation, or, more general, chemical reaction with surrounding media.

2) In many cases, very small clusters interact strongly with biological matter; e.g., Ag+DNA. Here, the system cluster+biomolecule becomes the interesting entity in its own right and needs to be studied (and applied) as such.

3) Even if there is no chemical bonding prevalent at the NP-matrix interface, the wave functions of 
the optically excited states can extend into the substrate, as shown e.g., for a $\mathrm{Au}_{20}$ cluster on $\mathrm{TiO}_{2}$ [58]. This has necessarily consequences for the optical properties but is not well understood.

4) Even without chemical bonding and quantum-mechanical interaction, the presence of the polarizable matrix influences, e.g., the LSPR energy, which is described already by classical Mie theory [3, 7]. But even if the interactions are very weak, as in the case of clusters embedded in rare-gas matrices, the crystal-field splitting is enough to be clearly visible in the optical spectra. The electrostatic model of solvation and the well known conductor-like screening model of solvation (COSMO), have recently been used in TDDFT calculations to describe the rare gas matrix [59] but are certainly inadequate for stronger interactions.

\subsection{Examples}

To finish, we present two examples which highlight the power of the TDDFT methods. Fig. 4 shows the absorption spectra of the ubiquitous icosahedral gold clusters compound $\mathrm{Au}_{144}(\mathrm{SR})_{60}$. The rather large calculation yields spectra that reproduce all the essential features of the measurement: all the peaks are present, although their height is somewhat overestimated. The only region which is not well represented is between $2.1 \mathrm{eV}$ and $2.5 \mathrm{eV}$. This comparison is one of the very few in this size range which will allow for direct comparisons with theory, thus providing the badly needed benchmark result needed to improve the choice of the approximations within TDDFT.

Fig. 5 shows the change of absorption spectra upon alloying silver clusters with gold, which in this size range does not show the LSPR. Note the agreement with the measurements in the inset.

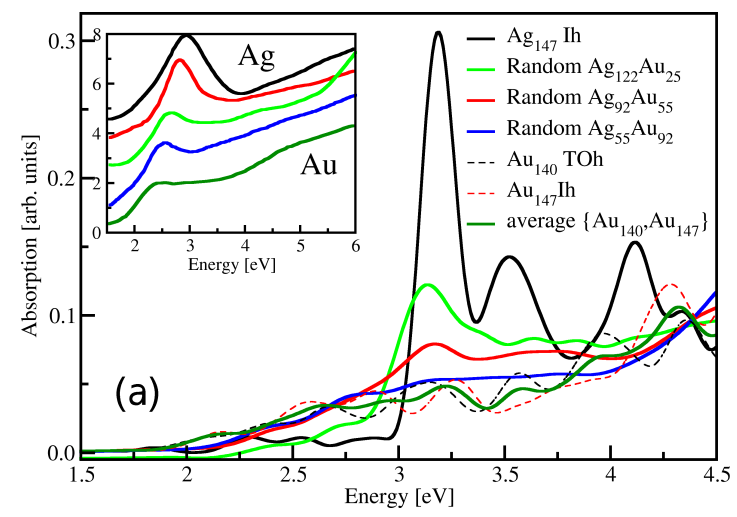

Figure 5 - Absorption spectra of 147-atom icosahedral Ag-Au random alloy clusters, compared with the experimental results of Gaudry et al. [60] shown in the inset for compositions $\mathrm{x}=0.00$, $0.25,0.50,0.75$, and 1.00. Figure taken from [61].

\section{Further Reading}

- Carsten A. Ullrich. Time-Dependent Density-Functional Theory: Concepts and Applications. Oxford University Press, 2011.

- G. Onida, L. Reining, and A. Rubio. Electronic excitations: density-functional versus many-body Green's-function approaches. Rev. Mod. Phys., 74:601, 2002. and references therein.

- E. Cottancin, G. Celep, J. Lermé, M. Pellarin, J. Huntzinger, J. Vialle, and M. Broyer. Optical properties of noble metal clusters as a function of the size: Comparison between experiments and a semi-quantal theory. Theoretical Chemistry Accounts: Theory, Computation, and Modeling (Theoretica Chimica Acta), 116:514, 2006.

- R. Ferrando, J. Jellinek, and R. L. Johnston. Nanoalloys: From theory to applications of alloys clusters and nanoparticles. Chem. Rev., 108:845-910, 2008.

- F. Baletto, R. Ferrando, A. Fortunelli, F. Montalenti, and C. Mottet. Crossover among structural motifs in transition and noble-metal clusters. J. Chem. Phys., 116:3856-3863, 2002. 
- Rongchao Jin, Chenjie Zeng, Meng Zhou, and Yuxiang Chen. Atomically precise colloidal metal nanoclusters and nanoparticles: Fundamentals and opportunities. Chemical Reviews, 116(18):10346-10413, 2016.

- F. Rabilloud. Assessment of the performance of long-range corrected density functionals for calculating the absorption spectra of silver clusters. J. Phys. Chem. A, 177:4267-4278, 2013.

- Hellmut Haberland. Looking from both sides. Nature, 494:E1-E2, 2013.

- U. Kreibig and M. Vollmer. Optical Properties of Metal Clusters. Springer-Verlag, Berlin, Heidelberg, New York, 1995.

- Xavier Andrade, David Strubbe, Umberto De Giovannini, Ask Hjorth Larsen, Micael J. T. Oliveira, Joseba Alberdi-Rodriguez, Alejandro Varas, Iris Theophilou, Nicole Helbig, Matthieu J. Verstraete, Lorenzo Stella, Fernando Nogueira, Alan Aspuru-Guzik, Alberto Castro, Miguel A. L. Marques, and Angel Rubio. Real-space grids and the octopus code as tools for the development of new simulation approaches for electronic systems. Phys. Chem. Chem. Phys., 17:31371-31396, 2015.

- Francesco Sottile, Fabien Bruneval, AG Marinopoulos, Luise Dash, Silvana Botti, Valerio Olevano, Nathalie Vast, Angel Rubio, and Lucia Reining. TDDFT from molecules to solids: The role of long-range interactions. International Journal of Quantum Chemistry, 102:684, 2005.

- Amendra Fernando, K. L. Dimuthu M. Weerawardene, Natalia V. Karimova, and Christine M. Aikens. Quantum mechanical studies of large metal, metal oxide, and metal chalcogenide nanoparticles and clusters. Chemical Reviews, 115(12):6112-6216, 2015. PMID: 25898274. 


\section{References}

\section{References}

[1] Ian Freestone, Nigel Meeks, Margaret Sax, and Catherine Higgitt. The lycurgus cup - a roman nanotechnology. Gold Bulletin, 40(4):270-277, Dec 2007.

[2] E. Cottancin, G. Celep, J. Lermé, M. Pellarin, J. Huntzinger, J. Vialle, and M. Broyer. Optical properties of noble metal clusters as a function of the size: Comparison between experiments and a semi-quantal theory. Theoretical Chemistry Accounts: Theory, Computation, and Modeling (Theoretica Chimica Acta), 116:514, 2006.

[3] G Mie. Beiträge zur Optik trüber Medien, speziell kolloidaler Metallösungen. Annalen der Physik, 330(3):377-445, 1908.

[4] Hans-Christian Weissker and Xochitl Lopez-Lozano. Surface plasmons in quantum-sized noble-metal clusters: Tddft quantum calculations and the classical picture of charge oscillations. Phys. Chem. Chem. Phys., 17:28379-28386, 2015.

[5] Walt A. de Heer. The physics of simple metal clusters: experimental aspects and simple models. Rev. Mod. Phys., 65:611-676, Jul 1993.

[6] M. Brack. The physics of simple metal clusters: self-consistent jellium model and semiclassical approaches. Rev. Mod. Phys., 65:677, 1993.

[7] U. Kreibig and M. Vollmer. Optical Properties of Metal Clusters. Springer-Verlag, Berlin, Heidelberg, New York, 1995.

[8] H.-Ch. Weissker and C. Mottet. Optical properties of pure and core-shell noble-metal nanoclusters from tddft: The influence of the atomic structure. Phys. Rev. B, 84:165443, Oct 2011.

[9] Rajarshi Sinha-Roy, Pablo Garcá-González, Hans-Christian Weissker, Frank Rabilloud, and Antonio I. Fernández-Domínguez. Classical and ab initio plasmonics meet at sub-nanometric noble metal rods. ACS Photonics, 4(6):1484-1493, 2017.

[10] H.-Ch. Weissker, H. Barron Escobar, V. D. Thanthirige, K. Kwak, D. Lee, G. Ramakrishna, R.L Whetten, and X. López-Lozano. Information on quantum states pervades the visible spectrum of the ubiquitous Au144 gold nanocluster. Nature Communications, DOI: 10.1038/ncomms4785, 2014.

[11] Sami Malola, Lauri Lehtovaara, Jussi Enkovaara, and Hannu Häkkinen. Birth of the localized surface plasmon resonance in monolayer-protected gold nanoclusters. ACS Nano, 7(11):10263-10270, 2013.

[12] Xóchitl Lopez-Lozano, Hector Barron, Christine Mottet, and Hans-Christian Weissker. Aspect-ratioand size-dependent emergence of the surface-plasmon resonance in gold nanorods - an ab initio tddft study. Phys. Chem. Chem. Phys., 16:1820-1823, 2014.

[13] Hellmut Haberland. Looking from both sides. Nature, 494:E1-E2, 2013.

[14] R Fuchs and F Claro. Multipolar response of small metallic sphere: Nonlocal theory. Physical Review B, 35(8):3722-3727, March 1987.

[15] F Javier García de Abajo. Nonlocal Effects in the Plasmons of Strongly Interacting Nanoparticles, Dimers, and Waveguides. J. Phys. Chem. C, 112(46):17983-17987, 2008.

[16] P Nordlander R Esteban, A G Borisov and J Aizpurua. Bridging quantum and classical plasmonics with a quantum-corrected model. Nature Comm., 8:825, 2012.

[17] W. Ekardt. Size-dependent photoabsorption and photoemission of small metal particles. Phys. Rev. $B, 31: 6360-6370,1985$.

[18] Amendra Fernando, K. L. Dimuthu M. Weerawardene, Natalia V. Karimova, and Christine M. Aikens. Quantum mechanical studies of large metal, metal oxide, and metal chalcogenide nanoparticles and clusters. Chemical Reviews, 115(12):6112-6216, 2015. PMID: 25898274. 
[19] Xavier Andrade, David Strubbe, Umberto De Giovannini, Ask Hjorth Larsen, Micael J. T. Oliveira, Joseba Alberdi-Rodriguez, Alejandro Varas, Iris Theophilou, Nicole Helbig, Matthieu J. Verstraete, Lorenzo Stella, Fernando Nogueira, Alan Aspuru-Guzik, Alberto Castro, Miguel A. L. Marques, and Angel Rubio. Real-space grids and the octopus code as tools for the development of new simulation approaches for electronic systems. Phys. Chem. Chem. Phys., 17:31371-31396, 2015.

[20] G. te Velde, F.M. Bickelhaupt, E.J. Baerends, C. Fonseca Guerra, S.J.A. van Gisbergen, J.G. Snijders, and T. Ziegler. Chemistry with adf. J. Comput. Chem., 22:931-967, 2001.

[21] https://wiki.fysik.dtu.dk/gpaw/.

[22] Walt A. de Heer. The physics of simple metal clusters: experimental aspects and simple models. Rev. Mod. Phys., 65:611, 1993.

[23] Rongchao Jin, Chenjie Zeng, Meng Zhou, and Yuxiang Chen. Atomically precise colloidal metal nanoclusters and nanoparticles: Fundamentals and opportunities. Chemical Reviews, 116(18):1034610413, 2016.

[24] Jonathan A. Scholl, Ai Leen Koh, and Jennifer A. Dionne. Quantum plasmon resonances of individual metallic nanoparticles. Nature, 483:421-427, 2012.

[25] N. Del Fatti, D. Christofilos, F. Vallée, and Dimitris Christofilos. Optical response of a single gold nanoparticle. Gold Bulletin, 41(2):147-158, Jun 2008.

[26] Murilo L. Tiago, Juan C. Idrobo, Serdar Öğüt, Julius Jellinek, and James R. Chelikowsky. Electronic and optical excitations in $a g_{n}$ clusters $(n=1--8)$ : Comparison of density-functional and manybody theories. Phys. Rev. B, 79:155419, 2009.

[27] G. Onida, L. Reining, and A. Rubio. Electronic excitations: density-functional versus many-body Green's-function approaches. Rev. Mod. Phys., 74:601, 2002. and references therein.

[28] Erich Runge and E.K.U. Gross. Density-Functional Theory for Time-Dependent Systems. Phys. Rev. Lett., 52:997, 1984.

[29] Carsten A. Ullrich. Time-Dependent Density-Functional Theory: Concepts and Applications. Oxford University Press, 2011.

[30] M. E. Casida. pages 155-192. World Scientific, 1995.

[31] K. Yabana and G. F. Bertsch. Time-dependent local-density approximation in real time. Phys. Rev. B, 54:4484-4487, Aug 1996.

[32] Olga Lopez-Acevedo, Hironori Tsunoyama, Tatsuya Tsukuda, Hannu Häkkinen , and Christine M. Aikens. Chirality and electronic structure of the thiolate-protected au38 nanocluster. Journal of the American Chemical Society, 132(23):8210-8218, 2010. PMID: 20499877.

[33] Emily Townsend and Garnett W. Bryant. Plasmonic properties of metallic nanoparticles: The effects of size quantization. Nano Letters, 12(1):429-434, 2012.

[34] T. P. Rossi, M. Kuisma, M. J. Puska, R. M. Nieminen, and P. Erhart. Kohn-Sham decomposition in real-time time-dependent density-functional theory: An efficient tool for analyzing plasmonic excitations. ArXiv e-prints, March 2017.

[35] Masashi Noda, Kazuya Ishimura, Katsuyuki Nobusada, Kazuhiro Yabana, and Taisuke Boku. Massively-parallel electron dynamics calculations in real-time and real-space: Toward applications to nanostructures of more than ten-nanometers in size. Journal of Computational Physics, 265:145 $-155,2014$.

[36] Oscar Baseggio, Martina De Vetta, Giovanna Fronzoni, Mauro Stener, and Alessandro Fortunelli. A new time-dependent density-functional method for molecular plasmonics: Formalism, implementation, and the au144(sh)60 case study. International Journal of Quantum Chemistry, 116(21):1603$1611,2016$.

[37] Hongping Xiang, Xu Zhang, Daniel Neuhauser, and Gang Lu. Size-dependent plasmonic resonances from large-scale quantum simulations. The Journal of Physical Chemistry Letters, 5(7):1163-1169, 2014. 
[38] R. Ferrando, J. Jellinek, and R. L. Johnston. Nanoalloys: From theory to applications of alloys clusters and nanoparticles. Chem. Rev., 108:845-910, 2008.

[39] F. Baletto, R. Ferrando, A. Fortunelli, F. Montalenti, and C. Mottet. Crossover among structural motifs in transition and noble-metal clusters. J. Chem. Phys., 116:3856-3863, 2002.

[40] Jack B. A. Davis, Sarah L. Horswell, and Roy L. Johnston. Application of a parallel genetic algorithm to the global optimization of gas-phase and supported gold-iridium sub-nanoalloys. The Journal of Physical Chemistry C, 120(7):3759-3765, 2016.

[41] Francesco Sottile, Fabien Bruneval, AG Marinopoulos, Luise Dash, Silvana Botti, Valerio Olevano, Nathalie Vast, Angel Rubio, and Lucia Reining. TDDFT from molecules to solids: The role of long-range interactions. International Journal of Quantum Chemistry, 102:684, 2005.

[42] R. van Leeuwen and E. J. Baerends. Exchange-correlation potential with correct asymptotic behavior. Phys. Rev. A, 49:2421, 1994.

[43] P. R. T. Schipper, O. V. Gritsenko, S. J. A. van Gisbergen, and E. J. Baerends. Molecular calculations of excitation energies and (hyper)polarizabilities with a statistical average of orbital model exchangecorrelation potentials. J. Chem. Phys., 112:1344, 2000.

[44] T. Leininger, H. Stoll, H. J. Werner, and A. Savin. Combining long-range configuration interaction with short-range density functionals. Chem. Phys. Lett., 275:151-160, 1997.

[45] F. Rabilloud. Assessment of the performance of long-range corrected density functionals for calculating the absorption spectra of silver clusters. J. Phys. Chem. A, 177:4267-4278, 2013.

[46] M. A. L. Marques, Alberto Castro, and Angel Rubio. Assessment of exchange-correlation functionals for the calculation of dynamical properties of small clusters in time-dependent density functional theory. The Journal of Chemical Physics, 115(7):3006-3014, 2001.

[47] Miguel A.L. Marques, Micael J.T. Oliveira, and Tobias Burnus. Libxc: A library of exchange and correlation functionals for density functional theory. Computer Physics Communications, 183(10):2272 $-2281,2012$.

[48] Hisayoshi Iikura, Takao Tsuneda, Takeshi Yanai, and Kimihiko Hirao. A long-range correction scheme for generalized-gradient-approximation exchange functionals. The Journal of Chemical Physics, 115(8):3540-3544, 2001.

[49] G.F. Zhao, Y. Lei, and Z. Zeng. Absorption spectra of small silver clusters agn $(\mathrm{n}=4,6,8)$ : A tddft study. Chem. Phys., 327:261, 2006.

[50] A. Castro, M.A.L. Marques, J.A. Alonso, A. Rubio, J. Zhao, and R.-H. Xie. Optical properties of nanostructures from time-dependent density functional theory. Journal of Computational and Theoretical Nanoscience, 1:231, 2004.

[51] D.R. Hamann. Generalized norm-conserving pseudopotentials. Phys. Rev. B, 40:2980, 1989.

[52] Eleonora Luppi, H.-Ch Weissker, Sandro Bottaro, Francesco Sottile, Valérie Veniard, Lucia Reining, and Giovanni Onida. Phys. Rev. B, 78(24):245124, 2008.

[53] P. E. Blöchl. Projector augmented-wave method. Phys. Rev. B, 50:17953, 1994.

[54] B. Anak, M. Bencharif, and F. Rabilloud. Time-dependent density functional study of uv-visible absorption spectra of small noble metal clusters $\left(\mathrm{Cu}_{n}, \mathrm{Ag}_{n}, \mathrm{Au}_{n}, n=2-9,20\right)$. RSC Adv., 4:1300113011, 2014.

[55] Alberto Castro, Miguel A. L. Marques, Aldo H. Romero, Micael J. T. Oliveira, and Angel Rubio. The role of dimensionality on the quenching of spin-orbit effects in the optics of gold nanostructures. The Journal of Chemical Physics, 129(14):144100, 2008.

[56] Andrea Marini. Ab Initio finite-temperature excitons. Phys. Rev. Lett., 101:106405, Sep 2008.

[57] Marie Lopez del Puerto, Murilo L. Tiago, and James R. Chelikowsky. Ab initio calculation of temperature effects in the optical response of open-shell sodium clusters. The Journal of Chemical Physics, 127(14):144311, 2007. 
[58] Run Long and Oleg V Prezhdo. Instantaneous generation of charge-separated state on tio2 surface sensitized with plasmonic nanoparticles. Journal of the American Chemical Society, 136(11):43434354, 2014. PMID: 24568726.

[59] L. Jensen, L. L. Zhao, and G. C. Schatz. Size-dependence of the enhanced raman scattering of pyridine adsorbed on $\operatorname{Ag}_{n}(n=2-8,20)$ clusters. J. Phys. Chem. C, 111:4756-4764, 2007.

[60] M. Gaudry, J. Lermé, E. Cottancin, M. Pellarin, J. L. Vialle, M. Broyer, B. Prével, M. Treilleux, and P. Mélinon. Optical properties of $\left(a u_{x} a g_{1-x}\right)_{n}$ clusters embedded in alumina: Evolution with size and stoichiometry. Phys. Rev. B, 64:085407, 2001.

[61] Xochitl López-Lozano, C. Mottet, and H .-Ch. Weissker. Effect of alloying on the optical properties of ag/au nanoparticles. The Journal of Physical Chemistry C, 117(6):3062-3068, 2013. 